\title{
Complex cognition: the science of human reasoning, problem-solving, and decision-making
}

\author{
Markus Knauff • Ann G. Wolf
}

Received: 10 March 2010/ Accepted: 10 March 2010/Published online: 23 March 2010

(C) Marta Olivetti Belardinelli and Springer-Verlag 2010

Climate change, globalization, policy of peace, and financial market crises-often we are faced with very complex problems. In order to tackle these complex problems, the responsible people should first come to mutual terms. An additional challenge is that typically the involved parties have different (often conflicting) interests and relate the problems to different emotions and wishes. These factors certainly do not ease the quest for a solution to these complex problems.

It is needless to say that the big problems of our time are not easy to solve. Less clear, however, is identifying the causes that led to these problems. Interest conflicts between social groups, the economic and social system or greedone can think of many responsible factors for the largescale problems we are currently confronted with.

The present "Special Corner: complex cognition" deals with questions in this regard that have often received little consideration. Under the headline "complex cognition", we summarize mental activities such as thinking, reasoning, problem-solving, and decision-making that typically rely on the combination and interaction of more elementary processes such as perception, learning, memory, emotion, etc. (cf. Sternberg and Ben-Zeev 2001). However, even though complex cognition relies on these elementary functions, the scope of complex cognition research goes beyond the isolated analysis of such elementary mental processes. Two aspects are essential for "complex cognition": The first aspect refers to the interaction of different mental activities such as perception, memory, learning,

M. Knauff $(\bowtie) \cdot$ A. G. Wolf

University of Giessen, Giessen, Germany

e-mail: Markus.Knauff@psychol.uni-giessen.de reasoning, emotion, etc. The second aspect takes the complexity of the situation into account an agent is confronted with. Based on these two aspects, the term "complex cognition" can be defined in the following way:

- Complex psychological processes: We talk about "complex cognition", when thinking, problem-solving, or decision-making falls back on other cognitive processes such as "perception", "working memory", "long-term memory", "executive processes", or when the cognitive processes are in close connection with other processes such as "emotion" and "motivation". The complexity also results from an interaction from a multitude of processes that occur simultaneously or at different points in time and can be realized in different cognitive and/or neuronal structures.

- Complex conditions: We also talk about "complex cognition" when the conditions are complex in which a person finds himself and in which conclusions need to be drawn, a problem needs to be solved, or decisions need to be made. The complexity of the conditions or constraints can have different causes. The situation structure itself can be difficult to "see", or the action alternatives are difficult "to put into effect". The conditions can themselves comprise of many different variables. These variables can exhibit a high level of interdependence and cross-connection, and it can, as time passes by, come to a change of the original conditions (e.g. Dörner and Wearing 1995; Osman 2010). It can also be the case that the problem is embedded in a larger social context and can be solved only under certain specifications (norms, data, legislations, culture, etc.) or that the problem can only be solved in interaction with other agents, be it other persons or technical systems. 
When one summarizes these two aspects, this yields the following view of what should be understood as "complex cognition".

As "complex cognition" we define all mental processes that are used by individuals for deriving new information out of given information, with the intention to solve problems, make decision, and plan actions. The crucial characteristic of "complex cognition" is that it takes place under complex conditions in which a multitude of cognitive processes interact with one another or with other noncognitive processes.

The "Special Corner: complex cognition" deals with complex cognition from many different perspectives. The typical questions of all contributions are: Does the design of the human mind enable the necessary thinking skills to solve the truly complex problems we are faced with? Where lay the boundaries of our thinking skills? How do people derive at conclusions? What makes a problem a complex problem? How can we improve our skills to effectively solve problems and make sound judgements?

It is for sure too much to expect that the Special Corner answers these questions. If it were that easy, we would not be still searching for an answer. It is, however, our intention with the current collection of articles to bring to focus such questions to a larger extent than has been done so far.

An important starting point is the fact that people's skills to solve the most complex of all problems and to ponder about the most complex issues is often immensehumankind would not otherwise be there were she is now. Yet, on the other hand, it has become more clear in the past few years that often people drift away from what one would identify as "rational" (Kahneman 2003). People hardly ever adhere to that what the norms of logic, the probability calculus, or the mathematical decision theory state. For example, most people (and organizations) typically accept more losses for a potential high gain than would be the case if they were to take into account the rules of the probability theory. Similarly, they draw conclusions from received information in a way that is not according to the rules of logic. When people, for example, accept the rule "If it rains, then the street is wet", they most often conclude that when the street is wet, it must have rained. That, however, is incorrect from a logical perspective: perhaps a cleaning car just drove by. In psychology, two main views are traditionally put forward to explain how such deviations from the normative guidelines occur. One scientific stream is interested in how deviations from the normative models can be explained (Evans 2005; JohnsonLaird 2008; Knauff 2007; Reason 1990). According to this line of research, deviations are caused by the limitations of the human cognitive system. The other psychological stream puts forward as the main criticism that the deviations can actually be regarded as mistakes (Gigerenzer 2008). The deviations accordingly have a high value, because they are adjusted to the information structure of the environment (Gigerenzer et al. 1999). They have probably developed during evolution, because they could ensure survival as for example the specifications of formal logic (Hertwig and Herzog 2009). We, the editors of the special corner, are very pleased that we can offer an impression of this debate with the contributions from Marewski, Gaissmaier, and Gigerenzer and the commentaries to this contribution from Evans and Over. Added to this is a reply from Marewski, Gaissmaier, and Gigerenzer to the commentary from Evans and Over.

Another topic in the area of complex cognition can be best illustrated by means of the climate protection. To be successful in this area, the responsible actors have to consider a multitude of ecological, biological, geological, political, and economical factors, the basic conditions are constantly at change, and the intervention methods are not clear. Because the necessary information is not readily available for the person dealing with the problem, the person is forced to obtain the relevant information from other sources. Furthermore, intervention in the complex variable structure of the climate can trigger processes whose impact was likely not intended. Finally, the system will not "wait" for intervention of the actors but will change itself over time. The special corner is also concerned with thinking and problem-solving in such complex situations. The article by Funke gives an overview of the current state of research on this topic from the viewpoint of the author, in which several research areas are covered that have internationally not received much acknowledgement (but see, for example, Osman 2010).

Although most contributions to the special corner come from the area of psychology, the contribution by Ragni and Löffler illustrates that computer science can provide a valuable addition to the understanding of complex cognition. Computer science plays an important role in complex cognition. In general, computer science, which is used to investigate computational processes central to all research approaches, can be placed in a "computational theory of cognition" framework. This is true especially for the development of computational theories of complex cognitive processes. In many of our modern knowledge domains, the application of simulations and modelling has become a major part of the methods inventory. Simulations help forecast the weather and climate change, help govern traffic flow and help comprehend physical processes. Although modelling in these areas is a vastly established method, it has been very little applied in the area of human thinking (but see e.g. Anderson 1990; Gray 2007). However, exactly in the area of complex cognition, the method 
of cognitive modelling offers empirical research an additional methodological access to the description and explanation of complex cognitive processes. While the validity of psychological theories can be tested with the use of empirical research, cognitive models, with their internal coherence, make possible to test consistency and completeness (e.g. Schmid 2008). They will also lead to new hypotheses that will in turn be possible to test experimentally. The contribution of Ragni and Löffler demonstrates with the help of an interesting example, finding the optimal route, the usefulness of simulation and modelling in psychology.

A further problem in the area of complex cognition is that many problems are solvable only under certain social conditions (norms, values, laws, culture) or only in interaction with other actors (cf. Beller 2008). The article on deontic reasoning by Beller is concerned with this topic. Deontic reasoning is thinking about whether actions are forbidden or allowed, obligatory or not obligatory. Beller proposes that social norms, imposing constraints on individual actions, constitute the fundamental concept for deontic thinking and that people reason from such norms flexibly according to deontic core principles. The review paper shows how knowing what in a certain situation is allowed or forbidden can influence how people derive at conclusions.

The article of Waldmann, Meder, von Sydow, and Hagmayer is concerned with the important topic of causal reasoning. More specifically, the authors explore the interaction between category and causal induction in causal model learning. The paper is a good example of how experimental work in psychology can combine different research traditions that typically work quite isolated. The paper goes beyond a divide and conquers approach and shows that causal knowledge plays an important role in learning, categorization, perception, decision-making, problem-solving, and text comprehension. In each of these fields, separate theories have been developed to investigate the role of causal knowledge. The first author of the paper is internationally well known for his work on the role of causality in other cognitive functions, in particular in categorization and learning (e.g. Lagnado et al. 2007; Waldmann et al. 1995). In a number of experimental studies, Waldmann and his colleagues have shown that people when learning about causal relations do not simply form associations between causes and effects but make use of abstract prior assumptions about the underlying causal structure and functional form (Waldmann 2007).

We, the guest editors, are very pleased that we have the opportunity with this Special corner to make accessible the topic "complex cognition" to the interdisciplinary readership of Cognitive Processing. We predict a bright future for this topic. The research topic possesses high research relevance in the area of basic research for a multitude of disciplines, for example psychology, computer science, and neuroscience. In addition, this area forms a good foundation for an interdisciplinary cooperation.

A further important reason for the positive development of the area is that the relevance of the area goes beyond fundamental research. In that way, the results of the area can for example also contribute to better understanding of the possibilities and borders of human thinking, problemsolving, and decisions in politics, corporations, and economy. In the long term, it might even lead to practical directions on how to avoid "mistakes" and help us better understand the global challenges of our time-Climate change, globalization, financial market crises, etc.

We thank all the authors for their insightful and inspiring contributions, a multitude of reviewers for their help, the editor-in-chief Marta Olivetti Belardinelli that she gave us the opportunity to address this topic, and the editorial manager, Thomas Hünefeldt, for his support for accomplishing the Special Corner. We wish the readers of the Special Corner lots of fun with reading the contributions!

\section{References}

Anderson JR (1990) The adaptive character of thought. Erlbaum, Hillsdale

Beller S (2008) Deontic norms, deontic reasoning, and deontic conditionals. Think Reason 14:305-341

Dörner D, Wearing A (1995) Complex problem solving: toward a (computer-simulated) theory. In: Frensch PA, Funke J (eds) Complex problem solving: the European perspective. Lawrence Erlbaum Associates, Hillsdale, pp 65-99

Evans JSBT (2005) Deductive reasoning. In: Holyoak KJ, Morrison RG (eds) The Cambridge handbook of thinking and reasoning. Cambridge University Press, Cambridge, pp 169-184

Gigerenzer G (2008) Rationality for mortals: how people cope with uncertainty. Oxford University Press, Oxford

Gigerenzer G, Todd PM, The ABC Research Group (1999) Simple heuristics that make us smart. Oxford University Press, New York

Gray WD (2007) Integrated models of cognitive systems. Oxford University Press, Oxford

Hertwig R, Herzog SM (2009) Fast and frugal heuristics: tools of social rationality. Soc Cogn 27:661-698

Johnson-Laird PN (2008) Mental models and deductive reasoning. In: Rips L, Adler J (eds) Reasoning: studies in human inference and its foundations. Cambridge University Press, Cambridge, pp 206-222

Kahneman D (2003) A perspective on judgment and choice: mapping bounded rationality. Am Psychol 58:697-720

Knauff M (2007) How our brains reason logically. Topio 26:19-36

Lagnado DA, Waldmann MR, Hagmayer Y, Sloman SA (2007) Beyond covariation: cues to causal structure. In: Gopnik A, Schulz L (eds) Causal learning: psychology, philosophy, and computation. Oxford University Press, Oxford, pp 154-172

Osman M (2010) Controlling uncertainty: a review of human behavior in complex dynamic environments. Psychol Bull 136(1):65-86 
Reason J (1990) Human error. Cambridge University Press, Cambridge

Schmid U (2008) Cognition and AI. KI 08/1, Themenheft "Kognition', pp 5-7

Sternberg RJ, Ben-Zeev T (2001) Complex cognition: the psychology of human thought. Oxford University Press, New York
Waldmann MR (2007) Combining versus analyzing multiple causes: how domain assumptions and task context affect integration rules. Cogn Sci 31:233-256

Waldmann MR, Holyoak KJ, Fratianne A (1995) Causal models and the acquisition of category structure. J Exp Psychol Gen 124:181-206 